POLIBETÁNICA

Núm. 39, pp. 49-78. México, 2015

\title{
CIANOFITAS DE LOS SISTEMAS FLUVIO-LAGUNARES POM-ATASTA Y PALIZADA DEL ESTE, ADYACENTES A LA LAGUNA DE TÉRMINOS, CAMPECHE, MÉXICO
}

\section{CYANOPHYTA IN FLUVIO-LAGOON SYSTEMS POM-ATASTA AND PALIZADA DEL ESTE, LAGUNA DE TERMINOS, CAMPECHE, MEXICO}

\author{
R.E. Muciño-Márquez ${ }^{1}$, M.G. Figueroa-Torres ${ }^{1}$ y A. Aguirre-León ${ }^{2}$ \\ ${ }^{1}$ Laboratorio de Ficología, ${ }^{2}$ Laboratorio Ecología Costera y Pesquerías; Universidad \\ Autónoma Metropólitana Unidad Xochimilco. Calzada del Hueso 1100, col. Villa Quietud, \\ CP 04960. DF, México. Correo electrónico: mucinoelizabeth@gmail.com
}

\begin{abstract}
RESUMEN
Las cianofitas son un grupo muy diverso de microorganismos procariontes fotosintetizadores, con una amplia distribución en diferentes ambientes tanto terrestres como acuáticos. Algunas especies pueden formar florecimientos algales nocivos o tóxicos. El objetivo de este estudio consistió en identificar la composición de las especies de cianofitas en los sistemas fluvio-lagunares Pom-Atasta (PA) y Palizada del Este (PE). Se recolectaron muestras de agua tanto en superficie como medio fondo para cada laguna, en febrero del 2011, en 10 sitios utilizando una botella van Dorn. Se identificaron 21 taxones, de las cuales siete especies forman florecimientos algales como: Pseudanabaena catenata, P. limnetica, Anabaenopsis circularis, A. elenkinii, Aphanizomenon cf. ovalisporum, Ap. schindleri y Dolichospermum circinalis. Además, se reportan dos nuevos registros correspondientes a Aphanizomenon cf. ovalisporum y Ap. schindleri.
\end{abstract}

Palabras clave: cianofitas, florecimientos algales, sistemas fluvio-lagunares, México.

\section{ABSTRACT}

The cyanophyta are a diverse group prokaryotic photosynthetic microorganisms, distributed in different terrestrial and aquatic environments. Some species of cyanophyta, can form toxic or harmful algal bloom. The objective of this study was to identify the species composition of cyanophyta in fluvio-lagoon systems PomAtasta (PA) and Palizada del Este (PE). Water samples were collected in ten sampling sites in each lagoon, with a van Dorn bottle at the surface and in the middle of the water column, in February, 2011. Twenty one were identified, of which seven species are bloom-forming as: Pseudanabaena catenata, P. limnetica, Anabaenopsis circularis, A. elenkinii, Aphanizomenon cf. ovalisporum, Ap. schindleri and Dolichospermum circinalis. Also, two new records reported: Aphanizomenon $\mathrm{cf}$ ovalisporum y Ap. schindleri.

Key words: cyanophyta, algal blooms, fluvio-lagoon systems, Mexico. 


\section{INTRODUCCIÓN}

Las cianobacterias, Cyanophyta, Cyanoprokaryota o algas verde azules son organismos procariotas, cuyo principal modo de obtención de energía para su metabolismo es la fotosíntesis (Anagnostidis y Komárek, 1985). Son un grupo muy diverso distribuido en cuerpos de agua continentales, salobres y marinos, pudiendo presentarse en ambientes con condiciones extremas como termales, en zonas antárticas, además de ambientes terrestres con cierto grado de humedad. Se les puede encontrar, como organismos libres, adheridos a sustratos vegetales, animales e inorgánicos o formando parte del fitoplancton o del fitobento (Anagnostidis y Komárek, 1985; Whitton y Potts, 2000; Tomitani et al., 2006; Meichtry-Zaburlín et al., 2009). Las cianofitas, poseen la capacidad de fijar el nitrógeno atmosférico, presentan vesículas de gas, que les ayudan a flotar para mantenerse en la zona fótica, además de tener la capacidad para crecer y reproducirse bajo condiciones de luz extremas, entre otras características, lo que hace que resulten en un grupo competitivamente exitoso en comparación con otros organismos similares o con otras algas. Algunas especies de cianofitas, en ciertas condiciones ambientales como temperaturas elevadas, alta concentración de nutrientes, baja relación N:P, pH alcalino, y columna de agua estratificada, pueden formar florecimientos algales, que dependiendo de las especies, pueden ser nocivos o tóxicos (Chorus y Bartram, 1999; Namikoshi et al., 2003; Campos et al., 2005; Otaño, 2009). En aguas continentales, las cianofitas son el grupo de microalgas con mayor número de especies toxigénicas, productoras de cianotoxinas (Pérez et al., 2008). A nivel mundial se han mencionado numerosos casos de mortandad de ganado, aves y animales silvestres, por el efecto directo de las cianotoxinas contenidas en las cianofitas, ingeridas por estos animales (Carmichael y Falconer, 1993; Skulberg et al., 1993; Quesada-Corral, et al., 2006). En México, se han reportado muchos eventos de florecimientos de cianofitas en varios estados de la república mexicana, como: Baja California Sur, Jalisco, Michoacán, Veracruz, San Luis Potosí, Sinaloa, Querétaro, Guanajuato, Puebla, Oaxaca, Hidalgo y en el Estado de México (Cortés-Altamirano y Licea-Durán, 1999; Ramírez-García et al., 2004; Oliva-Martínez et al., 2008; Arzate-Cárdenas et al., 2010; Vasconcelos et al., 2010; Sánchez-Chávez et al., 2011; Berry et al., 2011; Gárate-Lizárraga y Muciño-Márquez 2012; Tomasini-Ortiz et al., 2012); así como en diferentes cuerpos de agua como el lago de Chapultepec, los canales de Xochimilco, Cuemanco y la presa Zimapán. Los florecimiento algales nocivos (FAN) o tóxicos de las cianofitas, afectan la calidad del agua, los recursos pesqueros, a los animales y al hombre. Producen excesiva biomasa e inhiben la capacidad fotosintética de otras microalgas por enturbiar el agua, producen toxinas, provocan olores desagradables en el agua, disminuye la concentración de oxígeno disuelto y por tanto, su disponibilidad para organismos consumidores e inhiben el crecimiento de otras especies del fitoplancton que sirven de alimento a los consumidores (Chorus y Bartram, 1999; Echenique y Aguilera, 2009). Considerando la importancia de las especies de cianofitas que pueden formar FAN, el objetivo de este estudio fue en identificar la composición de las especies de este grupo, en los sistemas fluvio-lagunares Pom-Atasta (PA) y Palizada del Este (PE), Campeche. 
Muciño-Márquez,R.E.etal.:Cianofitasdesistemasfluvio-lagunaresPom-AtastayPalizadadel Este,Lag.deTérminos,Camp.,Méx.

\section{MATERIAL Y MÉTODOS}

El sistema PA se encuentra en la porción occidental de la Laguna de Términos entre los $18^{\circ} 30^{\prime}$ y $18^{\circ} 35^{\prime} \mathrm{N}$ y los $91^{\circ} 50^{\prime}$ y $92^{\circ} 20^{\prime} \mathrm{W}$. Su ubicación geográfica queda comprendida dentro de la llanura costera del sur del Golfo de México, formada por los ríos Grijalva y Usumacinta (Gutiérrez-Estrada et al., 1982). Por otra parte, el sistema PE se localiza en la porción suroeste de la Laguna de Términos, entre los $18^{\circ} 29^{\prime} 13^{\prime \prime}$ y $18^{\circ} 29^{\prime} 04^{\prime \prime} \mathrm{N}$ y los 914'36" y 9151'31" W (figs. 1A-1C). Se realizó un muestreo de cianofitas en los sistemas fluvio-lagunares Pom-Atasta y Palizada del Este (figs. 1B y 1C), las muestras fueron obtenidas en 10 sitios para cada sistema, los días 12 y 13 de febrero del 2011, utilizando una botella van Dorn tanto para muestras de superficie como de medio fondo (hasta donde penetra la luz, establecido con un disco de Secchi), dando un total de 40 muestras las cuales se colocaron en frascos de 250 $\mathrm{ml}$ y se fijaron con acetato-lugol en una proporción de 100:1 respecto a la muestra.

Para la identificación de las cianofitas, se tomaron alícuotas de $2 \mathrm{ml}$, las cuales se depositaron en cámaras de Utermöhl (Hasle, 1978), y se revisaron en un microscopio invertido Olympus CK40 utilizando objetivos de 40X y 60X, para la obtención de las fotomicrografías se empleó una cámara SONY de 7.2 mega pixeles y se consultaron las obras de Prescott (1962); Komárek y Anagnostidis (1999, 2005); Komárek y Komárková (2003); Otaño (2009); Hašler y Poulíčková (2010); Cirés-Gómez y Quesada de Corral (2011) y Novelo (2011). Se consultó el sistema AlgaeBase para verificar los nombres taxonómicos aceptados (Guiry y Guiry, 2012).

\section{RESULTADOS}

\section{Clave de identificación de los Órdenes de Cianofitas encontrados}

1a. Cianofitas filamentosas

1b. Cianofitas no filamentosas (unicelulares o coloniales). Unicelulares o cenobiales (con pocas células, unidos por capas mucilaginosas); reproducción por división binaria en uno o más planos. Chroococcales 2a. Filamentos simples sin heterocistos ni acinetos; pueden estar envueltos en vainas; reproducción por división celular en un plano; formación de hormogonios Oscillatoriales

2b. Filamentos con falsas ramificaciones en los tricomas, pueden formar acinetos y tener o no heterocitos, son pluricelulares, se reproducen mediante hormogonios y presenta división celular en un plano Nostocales 


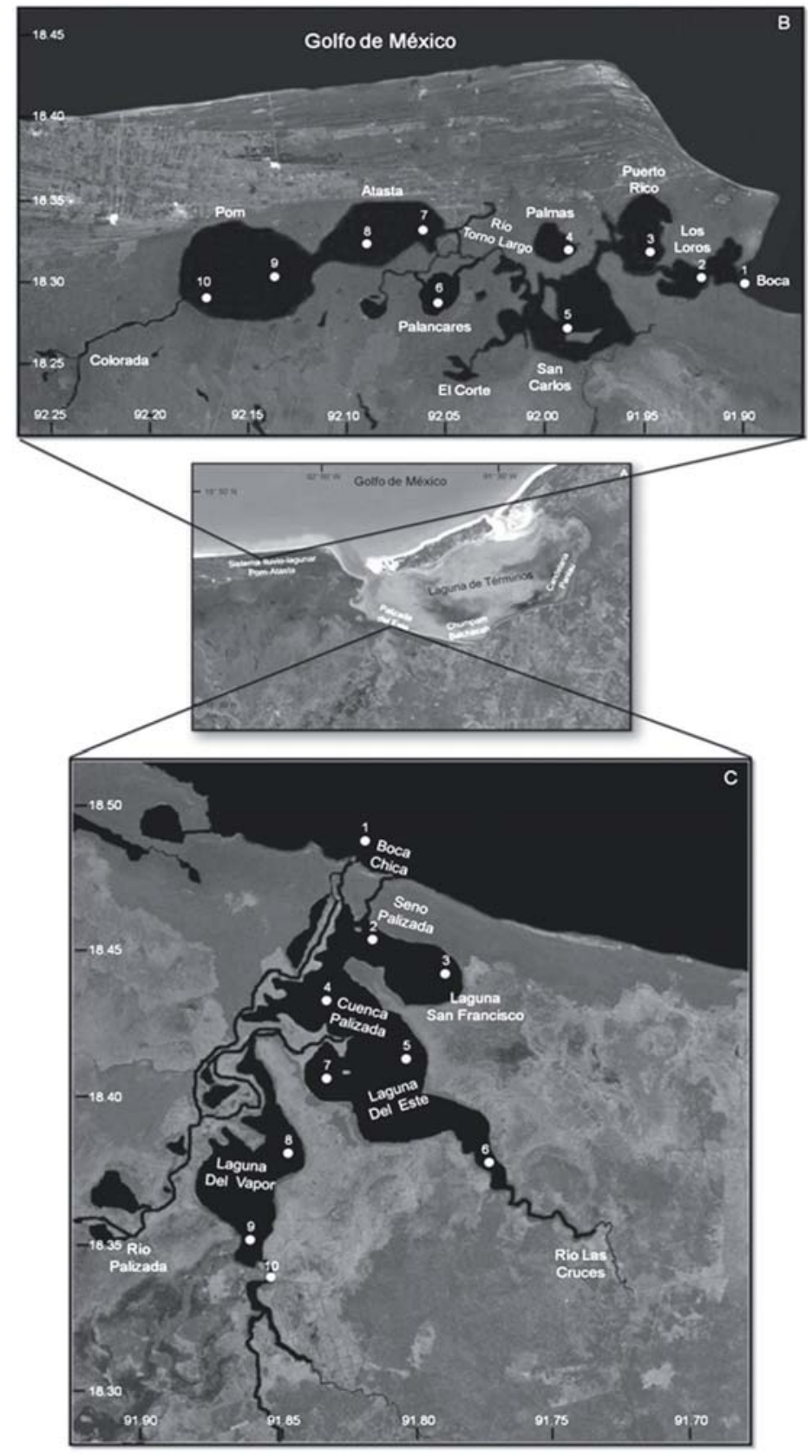

Fig. 1. (A) Localización geográfica de los sistemas fluvio-lagunares, (B) Pom-Atasta y (C) Palizada del Este. En el sur del Golfo de México. Se señala los sitios de muestreo (०). 


\section{Clave de identificación de géneros y especies del Órden Chroococcales}

1. Colonias compactas, células redondeadas, sin aerotopos, distribuidas irregularmente en el mucílago común. Células mayores de 1,5 a $3 \mu \mathrm{m}$ de diámetro, organizadas en colonias esféricas. . Aphanocapsa elachista 2. Colonias con pocas células (2 a 16), células generalmente segmentadas en la porción media. Chroococcus

2a. Células distribuidas irregularmente, mayores de 3.5-4.2 $\mu \mathrm{m}$ de diámetro

2b. Células con más de $5 \mu \mathrm{m}$ de diámetro C. dispersus 3. Colonias usualmente con un mayor número de células (40), células ovaladas forman grupos libres en el centro. Células de 6-12 $\mu \mathrm{m}$ de diámetro., la colonia rodeada de mucílago. Limnococcus limneticus

4. Colonias planas, células segmentadas en la porción media, distribuidas en el plano de la colonia en hileras perpendiculares formando una colonia tabular ..........Merismopedia 4a. Colonias rectangulares, células de 4-5 $\mu \mathrm{m}$ de diámetro................... convoluta 4b. Colonias cuadrangulares, células de $5 \mathrm{a} 6.5 \mu \mathrm{m}$ de diámetro ................. M. elegans 4c. Células dispuestas en filas paralelas, de 3-6.0 $\mu \mathrm{m}$ de diámetro.............. glauca 4d. Células menores de $3.5 \mu \mathrm{m}$ de diámetro.................................... punctata

\section{Aphanocapsa elachista West y G.S. West, 1894 Lám. 1, figs. 1 y 2}

Sinónimos: Microcystis pulverea f. elachista (West y G.S. West) Elenkin, 1938. M. elachista (West y G.S.West) Compère, 1967.

Descripción: colonias irregulares, difusas, mucílago fino, incoloro, hialino, las células dispersas, irregularmente distribuidas, células esféricas. Ancho de la colonia de 45.0 a $81.5 \mu \mathrm{m}$ y largo de 55 a $250 \mu \mathrm{m}$, con un diámetro de las células de 1.5 a $3 \mu \mathrm{m}$.

Distribución en el área de estudio: en los sistemas PA: lagunas de Palancares, Pom, y Atasta. PE: Boca Chica, entrada Seno Palizada Cuenca Palizada, en el río las Cruces y en las lagunas San Francisco, del Este y del Vapor. Abundancia de 35 a 175 x 103 cél•L $\mathrm{L}^{-1}$.

Distribución en el Golfo de México: reportada en el lago Mogo, región de los Tuxtlas, Veracruz (Vázquez y Blanco-Pérez, 2011); en la cuenca del río González, Tabasco (Moreno-Ruiz, 2005) y en la península de Yucatán (López-Adrián y Catzim-Cruz, 2010). 


\section{Chroococcus dispersus (Keissler) Lemmermann, 1904.}

Lám. 1, figs. 3 y 4.

Sinónimos: Chroococcus minor var. dispersus, von Keissler, 1902.

C. limneticus var. subsalsus, Lemmermann, 1901.

Gloeocapsa minor f. dispersa, (von Keissler) Hollerbach en Elenkin, 1938.

Descripción: colonias de 4 a 32 células, las células irregulares, las células son esféricas; con mucílago fino, hialino, incoloro, difuso, formando un margen indistinto. Diámetro de células de 3.5 a $4.2 \mu \mathrm{m}$.

Distribución en el área de estudio: en los sistemas PA: laguna de Pom. PE: Boca Chica, entrada Seno Palizada, cuenca Palizada, laguna del Este y río las Cruces. Abundancia de 2 a 24.5 x 103 cél $\bullet \mathrm{L}^{-1}$.

Distribución en el Golfo de México: reportada en el lago eutrófico el Chalchoapan, de San Martín Tuxtla, en el río Coatzacoalcos y cuenca del río Tonalá, Veracruz (Bozada y Páez, 1986; Pérez-Barrón, 1991; Vázquez et al., 2004; Moreno-Ruiz, 2005, Vázquez y Blanco-Pérez, 2011; Rodríguez-Palacio et al., 2008), en la cuenca del río González y laguna el Balsón, Tabasco (Moreno-Ruiz, 2005; Osorio-Sánchez y López-Pérez, 2005) y en la península de Yucatán (López-Adrián y Catzim-Cruz, 2010).

\section{Chroococcus minutus (Kützing) Nägeli, 1849. Lám. 1, figs. 5 y 6.}

Sinónimos: Protococcus minutus Kützing, 1843.

Chroococcus minutus var. minimus von Keissler, 1901.

Gloeocapsa minima (von Keissler) en Elenkin, 1938.

Chroococcus dispersus var. minor G.M. Smith, 1920.

Gloeocapsa minima f. smithii Hollerbach et al., 1953.

Descripción: colonias de 2 a 4 células, las células son ovaladas o esféricas. La colonia rodeada del mucílago fino, hialino, incoloro, difuso, formando un margen indistinto. Diámetro de le célula 5 a $9.5 \mu \mathrm{m}$.

Distribución en el área de estudio: en el sistema PE: entrada Seno Palizada y cuenca Palizada. Abundancia de $2 \times 103$ cél $\bullet \mathrm{L}^{-1}$.

Distribución en el Golfo de México: reportada en el río Sedeño y cuenca del río Tonalá, Veracruz (Licona, 2003; Moreno-Ruiz, 2005; Vázquez y Blanco-Pérez, 2011), en la cuenca del río González, Tabasco (Moreno-Ruiz, 2005) y en la península de Yucatán (López-Adrián y Catzim-Cruz, 2010). 
Muciño-Márquez,R.E.etal.: Cianofitasdesistemasfluvio-lagunaresPom-AtastayPalizadadel Este,Lag.deTérminos, Camp., Méx.

\section{Limnococcus limneticus (Lemmermann) Komárková, Jezberová, Komárek y} Zapomêlová, 2010. Lám. 1, figs. 7 y 8.

Sinónimos: Chroococcus limneticus Lemmermann, 1898. Gloeocapsa limnetica (Lemmermann) Hollerbach, 1938. Anacystis limnetica (Lemmermann) Drouet y Daily, 1952.

Descripción: colonias de 4 a 38 células, células ovaladas, rara vez esféricas o irregulares, las células son delgadas y distantes entre sí; la colonia rodeada del mucílago fino, hialino, incoloro, difuso, con un margen indistinto. Diámetro de la célula 6 a $12 \mu \mathrm{m}$.

Distribución en el área de estudio: en los sistemas PA: en la laguna de Pom. PE: Boca chica, entrada Seno Palizada y lagunas las Cruces, del Este, del Vapor y San Francisco. Abundancia de 3.5 a 17.5 x 103 cél $\bullet \mathrm{L}^{-1}$.

Distribución en el Golfo de México: reportada en lagos eutróficos alrededor de San Martín Tuxtla, lagos del Dique en Xalapa, río Sedeño, laguna de la Mancha y lago de Catemaco en Veracruz (Komárek y Komárková-Legnerová, 2002; Licona, 2003; Vázquez et al., 2004; Vázquez y Legaria-Moreno, 2006; Becerra-Cabañas, 2009; Vázquez y Blanco-Pérez, 2011), en la laguna el Balsón, Tabasco (Osorio-Sánchez y López-Pérez, 2005) y en la península de Yucatán (López-Adrián y Catzim-Cruz, 2010).

\section{Merismopedia convoluta Brébisson, en Kützing 1849.}

Lám. 1, figs. 9 y 10.

Sinónimos: Merismopedia willei Gardner, 1927.

Pseudoholopedia convoluta (Brébisson) Elenkin, 1938.

Descripción: colonias rectangulares, gran parte de la colonia están poco alineadas, distancia entre las células $4 \mu \mathrm{m}$, en grupos de más de 16 células, ordenadas verticalmente. Las células son segmentadas en la porción media elipsoidales. Colonia rodeada de mucílago fino, hialino, con un diámetro de la célula 4-5 $\mu \mathrm{m}$.

Distribución en el área de estudio: en el sistema PE: entrada Seno Palizada y laguna del Este. Abundancia de 2 a 64 x 103 cél $\bullet \mathrm{L}^{-1}$.

Distribución en el Golfo de México: reportada en el lago Chalchoapan, región de los Tuxtlas y cuenca del río González en Veracruz (Moreno-Ruiz, 2005; Rodríguez-Palacio et al., 2008). 


\section{Merismopedia elegans A. Braun en Kützing, 1849. Lam. 1, figs. 11 y 12.}

Descripción: colonias más o menos cuadrangulares, subesféricas o elipsoidales. Las células dispuestas densamente en filas perpendiculares, rodeadas de mucílago fino y hialino. Diámetro de la célula 5 a $6.5 \mu \mathrm{m}$ y longitud de 5 a $8.5 \mu \mathrm{m}$.

Distribución en el área de estudio: en el sistema PA: laguna de los Loros. Abundancia de 8.5 x 103 cél $\bullet \mathrm{L}^{-1}$

Distribución en el Golfo de México: reportada en los lagos del Dique, Xalapa, en la Boca de la laguna Sontecomapan y laguna de la Mancha, Veracruz (Vázquez y LegariaMoreno, 2006; Becerra-Cabañas, 2009; Muciño-Márquez et al., 2011). En la península de Yucatán, en Puerto Sisal y Dzilam (Herrera-Silveira y Morales-Ojeda, 2009; LópezAdrián y Catzim-Cruz, 2010).

\section{Merismopedia glauca (Ehrenberg) Kützing, 1845. \\ Lám. 1, figs. 13 y 14.}

Sinónimos: Merismopedia aeruginea Brébisson, 1849.

$$
\text { M. nova Wood, } 1872 .
$$

Descripción: colonias con 16 a 64 células, ovaladas o hemisféricas. Células dispuestas en filas paralelas. Colonia rodeada de mucílago fino, hialino. Diámetro de la célula 3-6 $\mu \mathrm{m}$. El mucílago tiene un margen de aproximadamente una célula de ancho.

Distribución en el área de estudio: en los sistemas PA: en la laguna las Palmas. PE: cuenca Palizada y laguna del vapor. Abundancia de 2 a 31 x 103 cél $\bullet \mathrm{L}^{-1}$.

Distribución en el Golfo de México: reportada en los ríos Chuniapan, Saltillo, Sedeño, Tecolapan, Xoteapan, San Joaquín en la Cascada de Matacapan, afluentes del río la Antigua, cuenca del río Tonalá, lago Majahual, lagos del Dique, Xalapa, Boca de la laguna Sontecomapan en Veracruz (Licona, 2003; Vázquez et al., 2004; Moreno-Ruiz, 2005; Villarauz, 2006; Becerra-Cabañas, 2009; Muciño-Márquez et al., 2011; Vázquez y Blanco-Pérez, 2011). En la península de Yucatán, en el Cenote Xmucuy, en los puertos Sisal, Chuburná, Progreso, Sacná, Telchac, Chabihau y Dzilam (López-Adrián y Barrientos-Medina, 2005; Herrera-Silveira y Morales-Ojeda, 2009, López-Adrián y Catzim-Cruz, 2010). 
Muciño-Márquez,R.E.etal.: Cianofitasdesistemasfluvio-lagunaresPom-AtastayPalizadadel Este,Lag.deTérminos, Camp., Méx.

\section{Merismopedia punctata Meyen, 1839.}

\section{Lám. 1, figs. 15 y 16.}

Sinónimos: Merismopedia kuetzingii Nägeli, 1849.

M. haumanii Kufferath, 1942.

Descripción: colonias cuadrangulares o rectangulares, muchas veces agrupadas en márgenes irregulares, entre 4-64 células, ovaladas. Colonia rodeada de mucílago fino, hialino. Diámetro de la célula 2.3-3.5 $\mu \mathrm{m}$. El mucílago tiene un margen de aproximadamente una célula de ancho.

Distribución en el área de estudio: en el sistema PE: laguna del Vapor. Abundancia de $32 \times 103$ cél $\bullet \mathrm{L}^{-1}$.

Distribución en el Golfo de México: reportada en el estuario del río Pánuco y la laguna de Pueblo Viejo en Tamaulipas (De la Lanza-Espino y Gómez-Rojas, 2005). En la cuenca del río González, cuenca baja del río Papaloapan, lago de Catemaco, lago Mongo y laguna de la Mancha, Veracruz (Komárková-Legnerová y Tavera, 1996, 2003; Komárek y Komárková-Legnerová, 2002; Legaria, 2003; Moreno-Ruiz, 2005; Vázquez y LegariaMoreno, 2006; Vázquez y Blanco-Pérez, 2011), así como también en la península de Yucatán (López-Adrián y Catzim-Cruz, 2010).

\section{Clave de identificación de géneros y especies del Órden Oscillatoriales}

1. Tricomas cilíndricos cortos, solitarios, rectos, o ligeramente curvos, células moniliformes

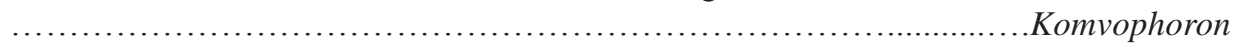

1a. Células intercalares cilíndricas, y célula apical redondeada ................... minutum

1b. Células forma de barril, células apicales moniliforme......................... sp.

2. Filamentos más o menos rectos, no atenuados apicalmente .........Phormidium retzii

3. Tricomas solitarios, rectos o espirados, vaina hialina delgada, células intercalares más o menos isodiamétricas, y apicales redondeadas ..............Planktolyngbya circumcreta 4. Tricomas en delicadas matas o a veces solitarios, con o sin aerotoposcélulas adyacentes unidas por un puente gelatinoso.......................................Pseudanabaena

4a. Célula apical convexa, la pared transversal reducida........................ catenata

4b. Célula apical suavemente redondeada en el extremo...................... limnetica 


\section{Komvophoron minutum (Skuja) Anagnostidis y Komárek, 1988.}

Lám. 2, figs. 1 y 2.

Sinónimo: Pseudanabaena minuta Skuja, 1948.

Descripción: tricomas cortos, flexuosos, ligeramente curvos, solitarios, aproximadamente con 30 células; células cilíndricas, margen cóncavo en la parte media, y célula apical redondeada. Ancho de las células de 2.5-3.5 $\mu \mathrm{m}$ y un largo de 3-5 $\mu \mathrm{m}$. Largo del tricoma de 38-100 $\mu \mathrm{m}$.

Distribución en el área de estudio: en los sistemas PA: laguna Loros. PE: las Cruces y laguna del Vapor. Abundancia de 15 x $103 \mathrm{cél} \bullet \mathrm{L}^{-1}$.

Distribución en el Golfo de México: primera vez que se reporta esta especie para el Golfo de México. El género se ha reportado en la laguna de Sontecomapan, Veracruz (Aké-Castillo y Vázquez, 2008; Okolodkov y Blanco Pérez, 2011) y en la península de Yucatán (López-Adrián y Catzim-Cruz, 2010).

\section{Komvophoron sp.}

Lám. 2, figs. 3 y 4.

Descripción: tricomas cortos, rectos, solitarios, aproximadamente con 25 células, rodeados de una capa mucilaginosa; las células con forma de barril, margen cóncavo en la parte media, y células apicales moniliforme o con verrugas (protuberancia). Ancho de las células de 4-5 $\mu \mathrm{m}$ y largo de 3-6 $\mu \mathrm{m}$. Largo del tricoma de 14-65 $\mu \mathrm{m}$.

Distribución en el área de estudio: en el sistema PE: laguna del Este y laguna del Vapor. Abundancia de 3 a 6 × 103 cél $\bullet \mathrm{L}^{-1}$.

\section{Phormidium retzii (C. Agardh) Kützing, en Gomont, 1892 \\ Lám. 2, figs. 5 y 6.}

Sinónimos: Oscillatoria retzii C. Agardh, 1812.

Conferva retzii (C. Agardh) Sommerfelt, 1826.

Phormidium retzii (C. Agardh) Kützing, 1843.

Microcoleus retzii (C. Agardh) Rabenhorst, 1847.

Lyngbya retzii (C. Agardh) Hansgirg, 1892.

Descripción: filamentos más o menos rectos, con vaina difluente. Tricomas no o ligeramente constrictos, sin gránulos en los septos. Células más cortas o más largas que anchas. Célula apical redondeada, generalmente sin engrosamiento apical, y sin caliptra. Ancho de las células de 5-11 $\mu \mathrm{m}$ y largo de 3.5-8 $\mu \mathrm{m}$; la célula apical con un largo de 7-9 $\mu \mathrm{m}$ y tricoma 9-88 $\mu \mathrm{m}$ de largo. 
Distribución en el área de estudio: En el sistema PE: cuenca Palizada y laguna del Vapor. Abundancia de $1.5 \times 103 \mathrm{cél} \bullet \mathrm{L}^{-1}$.

Distribución en el Golfo de México: reportada en la boca de laguna Sontecomapan, Veracruz (Muciño-Márquez et al., 2011).

Planktolyngbya circumcreta (G.S.West) Anagnostidis y Komárek, 1988. Lám. 2, fig. 7.

Sinónimo: Lyngbya circumcreta G.S.West, 1907.

Descripción: tricomas solitarios, desde irregularmente espiradis hasta levemente helicoidales con 2-2.5 vueltas, con vaina hialina. Las células intercalares son más o menos isodiamétricas, y las apicales redondeadas. Sin heterocito ni acinetos. Diámetro de la célula 2.5-3 $\mu \mathrm{m}$.

Distribución en el área de estudio: en el sistema PE: laguna del Vapor. Abundancia de 0.5 x 103 cél $\bullet L^{-1}$.

Distribución en el Golfo de México: reportada en el lago de Catemaco, Veracruz (Komárek y Komárková-Legnerová, 2002; Komárková-Legnerová y Tavera, 1996, 2003; Vázquez y Blanco-Pérez, 2011) y en la península de Yucatán (López-Adrián y Catzim-Cruz, 2010).

\section{Pseudanabaena catenata Lauterborn, 1915.}

Lám. 2, fig. 8.

Descripción: tricomas flexuosos, no atenuado hacia los extremos. Células cilíndricas, rectas, dolioliformes (en forma de barril) o ligeramente deprimidas en el centro con polos rectos o convexos y unidas entre sí por cordones intercelulares. Célula apical cilíndrica-arredondeada o levemente cónico-truncada, la pared transversal reducida, apareciendo casi rectangular cuando se ve de lado. Ancho de las células de 2-2.5 $\mu \mathrm{m}$ y largo de 2.5-4.5 $\mu \mathrm{m}$; largo del tricoma entre los 20-190 $\mu \mathrm{m}$.

Toxinas: produce hepatotoxinas, y forma florecimientos algales (Anagnostidis y Komarek, 1985; Lanzarot-Freudenthal, 2007).

Distribución en el área de estudio: en el sistema PE: entrada Seno Palizada, lagunas San Francisco y del Vapor. Abundancia de 4 a 80 x 103 cél $\bullet L^{-1}$.

Distribución en el Golfo de México: reportada en el lago Mongo, Veracruz (Vázquez y Blanco-Pérez, 2011), en el cenote Edzná, Campeche (López-Adrián y Barrientos-Medina, 2005) y en la península de Yucatán (López-Adrián y Catzim-Cruz, 2010). 


\section{Pseudanabaena limnetica (Lemmermann) Komárek, 1974. Lám. 2, fig. 9.}

Sinónimo: Oscillatoria limnetica Lemmermann, 1900.

Descripción: tricomas solitarios, ligeramente recto a curvado, sin aerótopos, sin vaina, con movimiento propio, células no atenuadas al final de los tricomas. Células vacuoladas, cilíndricas, constreñidas en las zonas de contacto. Ancho de las células de 1.3-1.8 $\mu \mathrm{m}$ y largo de 2-3.5 $\mu \mathrm{m}$; un largo del tricoma 12-180 $\mu \mathrm{m}$.

Toxinas: no produce toxinas, forma florecimientos algales y puede causar agotamiento de oxígeno en el agua (Chomérat et al., 2007).

Distribución en el área de estudio: en los sistemas PA: laguna Loros, laguna de San Carlos, laguna de Palancares, laguna de Atasta y laguna de Pom. PE: río las Cruces, laguna del Este y laguna del Vapor. Abundancia de 5.5 a 50 x 103 cél $\bullet^{-1}$.

Distribución en el Golfo de México: reportada para la cuenca del río González y lago Mongo en Veracruz (Moreno-Ruiz, 2005; Vázquez y Blanco-Pérez, 2011).

\section{Clave de identificación de géneros y especies del Órden Nostocales}

1. Tricomas con extremos atenuados o planos, con heterocistos........................

2a. Heterocistos en posición terminal..............................................

2b. Heterocistos intercalares................................................. 4

3a. Tricomas solitarios, no atenuados, inicialmente metaméricos pero con heterocistos en posición terminal tras la división del tricoma ..Anabaenopsis

$3 \mathrm{~b}$. Tricoma ligeramente arqueado, heterocistos terminales esféricos a ovalados A. circularis

3c. Tricomas circulares, heterocistos terminales esféricos y aerotopos presente

A. elenkinii

$3 \mathrm{~d}$. Tricomas solitarios, atenuados; heterocistos formados siempre a partir de células vegetativas terminales Cylindrospermopsis

3e. Tricomas en espiral o circulares, células apicales pequeñas, cónicas y por lo general ligeramente más estrecho y redondeados en el ápice............................ taverae 3f. Tricomas ligeramente recto, atenuados gradualmente en los extremos, célula apical cónicamente atenuada y puntiaguda, heterocisto en forma de gota............... c. cuspis 4a. Tricomas no atenuados, metaméricos, rectos o espirados; acinetos redondeados, subesféricos o elipsoidales, solitarios o en pares; célula con vesículas de gas. Heterocistos intercalares..... Anabaena 4b. Tricoma recto a ligeramente curvado, heterocistos cilíndricos intercalares....A. sp. $4 \mathrm{c}$. Tricomas agrupados en haces o colonias, gradual y ligeramente atenuados, células terminales alargadas y generalmente redondeadas o puntiagudas; acinetos de formas variadas, 
desde subesféricos a cilíndricos, en posición ligeramente alejados de los heterocistos que son intercalares Aphanizomenon 4d. Célula terminal elongada, heterocistos intercalares subesféricos, acineto ovalado A. cf. ovalisporum

4c. Célula terminal redondeada, heterocistos intercalares cilíndricos y acinetos también intercalares esféricos A. schindleri 5a. Tricoms solitarios, acinetos redondeados, aislados o en pares, próximos a un heterocisto, células con aerotopos Dolichospermum $5 b$. Tricomas circulares, células esféricas, heterocistos esféricos D. circinalis

\section{Anabaenopsis circularis (G.S.West) Wołoszyńska y Miller, 1923. Lám. 3. figs. 1 y 2.}

Sinónimos: Anabaena flos-aquae var. circularis G.S.WEST, 1907.

A. circularis (G.S.West) Wołoszyńska, 1912.

Anabaenopsis circularis (G.S.West) Wołoszyńska y Miller, 1923.

A. nadsonii sensu Proškina-Lavrenko y Makarova, 1968.

A. elenkinii f. circularis (G.S.West) Jeeji-Bai, 1977.

Descripción: tricomas ligeramente arqueados, en espiral o circulares, cortos, solitarios. Células cilíndricas y por lo general ligeramente arqueadas. Heterocistos esféricos a ovalados, se disponen habitualmente de dos en dos en cada extremo del filamento. Acinetos ovalados, se disponen a la mitad de los tricomas, separados de los heterocistos. Diámetro celular de 4-6.5 $\mu \mathrm{m}$ y largo de 4-9.5 $\mu \mathrm{m}$, diámetro del tricoma $10-55 \mu \mathrm{m}$; diámetro del los heterocistos redondos 5-7 $\mu \mathrm{m}$ y de los ovalados de 4-7deancho y5-7.5 $\mu \mathrm{m}$ de largo; y acinetos 8.5-11 $\mu \mathrm{m}$ de ancho y 4-7 $\mu \mathrm{m}$ de largo.

Toxinas: produce toxinas (Walker, 2004).

Distribución en el área de estudio: en el sistema PA: laguna las Palmas y laguna San Carlos. Abundancia de 10 x 103 cél $\bullet \mathrm{L}^{-1}$.

Distribución en el Golfo de México: reportada en los lagos eutróficos Chalchoapan, Verde y El Mogo y los cuerpos de agua mesotróficos Majahual y Manantiales, Veracruz (Vázquez et al., 2002; Vázquez et al., 2005; Vázquez y Blanco-Pérez, 2011) y en la cuenca del río González, Tabasco (Moreno-Ruiz, 2005).

\section{Anabaenopsis elenkinii V.V. Miller, 1923.}

\section{Lám. 3, figs. 3 y 4.}

Sinónimos: Anabaenopsis elenkinii f. curta Dedus-Ščegol, 1959.

Anabaenopsis elenkinii f. ovalispore Dedus-Ščegol, 1959. 
Descripción: tricomas circulares, cortos y solitarios, células cilíndricas con extremidades redondeadas, aerotopos presentes y heterocistos terminales esféricos. Diámetro celular de 4-6 $\mu \mathrm{m}$ y largo de 5-9 $\mu \mathrm{m}$; largo del tricoma 30-85 $\mu \mathrm{m}$, y heterocistos redondos con un diámetro de $3.5-6.5 \mu \mathrm{m}$.

Toxicidad: forma florecimientos algales nocivos o tóxicos (Alonso-Rodríguez y PáezOsuna, 2003; Alonso-Rodríguez et al., 2004; Komárek, 2005) y ha ocasionado disminución de oxígeno disuelto en estanques de cultivo de camarón (Cortés-Altamirano y Licea-Durán, 1999).

Distribución en el área de estudio: en el sistema PE: río las Cruces, lagunas del Este y del Vapor. Abundancia de 4.5 x 103 cél $\bullet L^{-1}$.

Distribución en el Golfo de México: reportada en el río Pánuco, laguna las Marismas y laguna de Pueblo Viejo, Tamaulipas (De la Lanza-Espino y Gómez-Rojas, 2005) y en el río Sedeño, Veracruz (Licona, 2003; Vázquez y Blanco-Pérez, 2011) como en la cuenca del río González y laguna el Balsón, Tabasco (Moreno-Ruiz, 2005; Osorio-Sánchez y López-Pérez, 2005).

\section{Anabaena sp.}

\section{Lám. 3, figs. 5 y 6.}

Descripción: tricoma solitrios recto a ligeramente curvado, con ligeras constricciones entre células, células en forma de barril a cilíndricas, generalmente elongadas. Célula terminal ligeramente más estrecha que las células intercalares. Heterocistos cilíndricos. Acinetos cilíndricos, claramente alargados y distantes de los heterocistos. Ancho celular de 2-3 $\mu \mathrm{m}$ y largo de 3-8.5 $\mu \mathrm{m}$, el tricoma con una longitud de $98 \mu \mathrm{m}$, ancho de los heterocistos de $3-4.5 \mu \mathrm{m}$ y largo de $4-7 \mu \mathrm{m}$ y ancho de acineto $5.5 \mu \mathrm{m}$ y largo de $25 \mu \mathrm{m}$.

Distribución en el área de estudio: en el sistema PA: laguna las Palmas. Abundancia de 1.5 x 103 cél $\bullet L^{-1}$.

\section{Aphanizomenon cf. ovalisporum Forti, 1911. Lám. 3, figs. 7 y 8 .}

Sinónimo: Anabaena ovalisporum Forti 1911.

Descripción: tricomas solitarios rectos, con constricciones entre células; células intercalares en forma de barril. Célula terminal elongada, ligeramente hialina, con el borde redondeado. Heterocistos subesféricos, acineto ovalado. Ancho celular de 4-6 $\mu \mathrm{m}$ y largo de 4.5-11 $\mu \mathrm{m}$, el tricoma con un largo de $110 \mu \mathrm{m}$, ancho de los heterocistos de 4.5-7.5 $\mu \mathrm{m}$ y largo de 3.5-11.5 $\mu \mathrm{m}$ y ancho del acineto $5.5-13 \mu \mathrm{m}$ y largo de $7.5-19 \mu \mathrm{m}$. 
Toxicidad: forma florecimientos nocivos y tóxicos, puede producir cilindrospermopsinas, saxitoxinas o anatoxinas (Pollingher et al., 1998; Banker et al., 2000; Quesada et al., 2006; Cirés-Gómez y Quesada de Corral, 2011).

Distribución en el área de estudio: rn el sistema PA: laguna San Carlos. Abundancia de $0.5 \times 103$ cél $\bullet \mathrm{L}^{-1}$.

Distribución en el Golfo de México: primera vez que se reporta en aguas mexicanas. Ha sido registrada en España (Álvarez-Cobelas y Gallardo, 1988, Quesada et al., 2006; Carrasco et al., 2007; Cirés-Gómez y Quesada de Corral, 2011), Polonia (Kokocinski y Soininen, 2012), Turquía (Ersanli y Gönülol, 2006), Israel (Banker et al., 2000; Leão et al., 2009), China (Wu et al., 2010), Australia y Nueva Zelanda (Bostock y Holland, 2010).

\section{Aphanizomenon schindleri Kling, Findlay y Komarek, 1994. \\ Lám. 3, figs. 9 y 10.}

Descripción: tricomas solitarios rectos, células intercalares cilíndricas, con vesículas de gas. Células terminales redondeadas. Heterocistos cilíndricos y acinetos esféricos intercalares. Largo del tricoma 110-600 $\mu \mathrm{m}$, ancho de las células de 3.5-4 $\mu \mathrm{m}$ y largo de 4-9 $\mu \mathrm{m}$, ancho de la célula terminal de 3-4 $\mu \mathrm{m}$ y largo de 7.5-11 $\mu \mathrm{m}$, ancho de los heterocistos 4-5.5 $\mu \mathrm{m}$ y largo de 4-4.8 $\mu \mathrm{m}$, ancho de acinetos 6-8.5 $\mu \mathrm{m}$ y largo de 9-15 $\mu \mathrm{m}$.

Toxinas: no producen toxinas. Forma florecimientos algales nocivos, produce mucílago, ocasionan mal olor en cuerpos de agua y sabor desagradable (Otaño, 2009).

Distribución en el área de estudio: en los sistemas PA: laguna Loros y laguna de Palancares. PE: río las Cruces y laguna del Vapor. Abundancia de 0.5 x 103 cél•L-1.

Distribución en el Golfo de México: primera vez que se reporta en aguas mexicanas. Ha sido reportada en Canadá y Argentina (Kling et al., 1994; Otaño, 2009).

\section{Cylindrospermopsis taverae Komárek y Komárková-Legnerová, 2002. Lám. 3, figs. 11 y 12.}

Descripción: tricomas solitarios en espirales o circulares, más o menos irregulares. Células cilíndricas o ligeramente en forma de barril, células apicales pequeñas, cónicas y por lo general ligeramente más estrechas y redondas en el ápice. En este estudio no se observaron heterocistos, Komárek y Komárková-Legnerová (2002) mencionan que el heterocisto es ligeramente alargado, cónico, curvado, y redondeado en el vértice. Diámetro celular de $1.5 \mu \mathrm{m}$ y largo de $2.8 \mu \mathrm{m}$.

Distribución en el área de estudio: en el sistema PE: laguna del Vapor y laguna del Este. Abundancia de $8 \times 103$ cél $\bullet L^{-1}$. 
Distribución en el Golfo de México: primera vez que se reporta para el Golfo de México. Se ha reportado en estanques eutróficos en San Luis Potosí (Komárek y KomárkováLegnerová, 2002).

\section{Cylindrospermopsis cuspis J.Komárek y H.Kling, 1991. Lám. 3, figs. 13 y 14.}

Descripción: tricomas solitarios, ligeramente rectos, atenuados gradualmente en los extremos. Células intercalares cilíndricas, más largas que anchas, las células apicales cónicas atenuadas, puntiagudas. Heterocisto en forma de gota, se desarrolla en un extremo del tricoma, a partir de las células apicales, son alargado-cónicos, con extremo puntiagudo. Ancho celular de 1-1.2 $\mu \mathrm{m}$ y largo de 4-16 $\mu \mathrm{m}$, un largo del tricoma de 40-160 $\mu \mathrm{m}$, y largo del heterocisto de6.5-10 $\mu \mathrm{m}$ y ancho de $1.2 \mu \mathrm{m}$.

Distribución en el área de estudio: en los sistemas PA: lagunas los Loros y las Palmas. PE: laguna del Vapor. Abundancia de 0.5 a 8.2 x 103 cél $\bullet \mathrm{L}^{-1}$.

Distribución en el Golfo de México: reportada en la región de los Tuxtlas, Veracruz (Komárek y Komárková-Legnerová, 2002, 2003).

\section{Dolichospermum circinalis (Rabenhorst ex Bornet y Flahault) Wacklin et al., 2009. Lám. 3, figs. 15 y 16.}

Sinónimos: Anabaena hassallii Witrock ex Lemmermann, 1907. A. circinalis Rabenhorst ex Bornet y Flahault, 1888.

Descripción: tricomas solitarios circulares, cortos, células intercalares esféricas, heterocistos esféricos. Komárek y Zapomělová (2007) reportan acinetos ovalados, únicos, raramente dos juntos y separados de los heterocitos. Diámetro celular de 8.0-13.5 $\mu \mathrm{m}$ y del heterocisto de 8-10 $\mu \mathrm{m}$ y largo del tricoma entre los 8-11 $\mu \mathrm{m}$.

Toxinas: forma florecimientos algales tóxicos, produce intoxicación paralizante por consumo de mariscos y saxitoxina (Mihali et al., 2009). Se ha reportado que diferentes poblaciones australianas son productoras de neurotoxinas y anatoxinas (Beltrán y Neilan, 2000).

Distribución en el área de estudio: en el sistema PE: Boca Chica, cuenca Palizada y laguna del Este. Abundancia de 500 a 1000 x 103 cél• $\mathrm{L}^{-1}$.

Distribución en el Golfo de México: reportada para la delta del río Mississippi y en las costas subregionales de Louisiana, Estados Unidos, (Bricker et al., 1997; Dortch, 2002) y en la península de Yucatán (López-Adrián y Catzim-Cruz, 2010). 
Muciño-Márquez,R.E.etal.:Cianofitasdesistemasfluvio-lagunaresPom-AtastayPalizadadel Este,Lag.deTérminos,Camp.,Méx.

\section{DISCUSIÓN}

Entre los años de 1965 a 1974 , se inician los estudios sobre la composición del fitoplancton en la laguna de Términos, abarcando la Boca del sistema Pom-Atasta y la Boca Chica del sistema Palizada del Este (Suárez-Cabro y Gómez-Aguirre, 1965; Gómez-Aguirre, 1974) y no se le da la continuidad. Éste es el primer trabajo que abarca el análisis de la composición, distribución y abundancia del fitoplancton en los cuerpos lagunares como tal de los dos sistemas estudiados, en particular de las cianofitas. Los resultados de este estudio aportan nuevo conocimiento sobre este grupo de microalgas que incluyó 21 taxones, pertenecientes a tres órdenes y doce géneros, de los cuales siete especies forman florecimientos algales: Pseudanabaena catenata, P. limnetica, Anabaenopsis circularis, A. elenkinii, Aphanizomenon cf. ovalisporum, Ap. schindleri y Dolichospermum circinalis, y de éstas cuatro son productoras de toxinas. Además, se registran para México por primera vez las especies Aphanizomenon cf. ovalisporum y Ap. schindleri, que forman florecimientos algales nocivos o tóxicos, causando mortandad de peces, provocando mal olor y sabor desagradables en el agua, además de la disminución en la concentración de oxígeno disuelto (Quesada et al., 2006; Otaño, 2009; Cirés-Gómez y Quesada de Corral, 2011).

De las especies identificadas en este estudio que forman FAN, se han reportado efectos negativos en cuerpos de agua, como es el caso de Anabaenopsis elenkinii en Sinaloa, que ocasionó un FAN en un estanque de cultivo de camarón, provocando la mortandad de peces y disminución de oxígeno di- suelto (Cortés-Altamirano y Licea-Durán, 1999; Alonso-Rodríguez y Páez-Osuna, 2003; Alonso-Rodríguez et al., 2004). Otros casos están relacionados con Dolichospermum circinalis en el Río Darling, Australia en 1991, formó un florecimiento algal neurotóxico, causando muerte de ganado (Bowling y Baker, 1996). Igualmente, el género Cylindrospermopsis incluye especies toxicas como $C$. raciborskii de distribución cosmopolita, que se ha reportado en la Laguna Asmolapan, Veracruz (Komárek y Komárková-Legnerová, 2002) y en la Península de Yucatán (López-Adrián y Catzim-Cruz, 2010). Esta es la especie que produce microcystina, cylindrospermopsina, saxitoxina y neosaxitoxina. Las primeras dos son hepatotóxicas mientras que las saxitoxinas son neurotóxicas (Hawkins et al., 1985; Carneiro et al., 2009). Otro caso reportado para C. raciborskii, la isla Palm en Australia, provocando la muerte de ganado bovino y enfermedades hepáticas en humanos, ya que produce espumas superficiales, mal olor y sabor en el agua (Jones y Sauter, 2005).

Una de las causas posibles de que las especies de cianofitas de este estudio no formaran florecimientos, puede deberse a la mezcla de agua marina y continental, que ocasiona una variación en la concentración de la salinidad. Madigan et al. (2004) han reportado que algunas microalgas son sensibles a los cambios bruscos en la concentración de la salinidad y no resisten los choques osmóticos en las células. Al parecer, las especies oportunistas formadoras de FAN registradas para los sistemas Pom-Atasta y Palizada del Este no tienen las condiciones óptimas para que estos florecimientos se desarrollen, los cuales podrían causar mortandad de animales, ya 
que son zonas pesqueras (Yáñez-Arancibia et al., 1980; Ayala-Pérez et al., 1998; Aguirre-León et al., 2010), e inclusive provocar intoxicaciones en las poblaciones aledañas. Otra posible explicación a la no formación de FAN es la temporada del estudio, ya que las colectas de las muestras sólo se realizaron en invierno, y los florecimientos nocivos de las cianofitas suelen darse en el verano, en esta temporada hay una disponibilidad de nutrientes, un aumento de temperatura, salinidad, $\mathrm{pH}$, una alta evaporación y la columna de agua se encuentra estratificada (Carvalho et al., 2008; Carvajal, 2011).

Por otra parte, es importante señalar que algunas especies de cianofitas son consideradas de gran utilidad como indicadores de estrés ambiental, ya que presentan una gran variedad de características ecofisiológicas que les permiten ser exitosas en cuerpos de agua eutróficos (Jozwiak et al., 2008). En el presente estudio se reportan cinco especies, Chroococcus minutus, Limnococcus limneticus, Merismopedia elegans, M. glauca y M. punctata que son indicadoras de aguas con impurezas moderada o contaminación moderada (Moreno-Ruiz, 2000; John et al., 2002).

La presencia de estas especies per se, nos indica la potencialidad de expresión de que en estos sistemas está ocurriendo algo, como la entrada de desechos urbanos, por lo cual que su presencia constituye un riesgo potencial frente a un aumento en la concentración de nutrientes y la incidencia de factores ambientales óptimos para que las especies oportunistas puedan formar FAN. Para prevenir una situación de vulnerabilidad ante la alteración por la presencia y posible desarrollo de especies tóxicas, se requieren monitorear estos sistemas. Por lo cual es importante, seguir realizando estudios de identificación y cuantificación de las cianofitas en estos ecosistemas, ya que brindan conocimiento de la ecología y la dinámica de los FAN y tóxicos y ayudan a prevenir en la salud de la población local. Futuros estudios aportaran información al respecto.

\section{AGRADECIMIENTOS}

Este estudio fue apoyado por la Universidad Autónoma Metropolitana-Xochimilco a través de los proyectos de investigación: Mareas rojas emergentes de México, y Ecología y Evaluación de las Comunidades de Peces en Sistemas Costeros de Veracruz y Campeche, Golfo de México y Sistemas Coralinos de Quintana Roo, Caribe Mexicano. UAM-X/CBS/El Hombre y Su Ambiente, al doctor Ismael Gárate-Lizárraga y a los revisores por las observaciones realizadas al manuscrito.

\section{LITERATURA CITADA}

Aguirre-León, A.; S. Díaz-Ruiz, y A.B. Ramírez-Huerta, 2010. Ecología de peces dominantes costeros tropicales. Estudio para el sistema fluvio-deltáico Pom-Atasta, Campeche. (Eds.). Universidad Autónoma Metropolitana Unidad Xochimilco, México. 136 pp.

Aké-Castillo, J.A., y G. Vázquez, 2008. "Phytoplankton variation and its relation to nutrients and allochthonous organic matter in a coastal lagoon on the Gulf of Mexico". Estuar. Coast. Shelf Sci., 78: 705-714. 
Muciño-Márquez,R.E.etal.:Cianofitasdesistemasfluvio-lagunaresPom-AtastayPalizadadel Este,Lag.deTérminos,Camp.,Méx.

Alonso-Rodríguez, R., y F. Páez-Osuna, 2003. "Nutrients, phytoplankton and harmful algal blooms in shrimp ponds: a review with special reference to the situation in the Gulf of California". Aquaculture, 219(1-4): 317-336.

Alonso-Rodríguez, R., F. Páez-Osuna, y I. Gárate-Lizárraga, 2004. El fitoplancton en la camaronicultura y larvicultura: importancia de un buen manejo. (Eds.). ICMyL-UNAM y CESASIN, México. 147 pp.

Álvarez-Cobelas, M., y T. Gallardo, 1988. "Catálogo de las algas continentales españolas V. Cyanophyceae Schaffner 1909". Act. Bot. Malacitana, 13: 53-76.

Anagnostidis, K., y J. Komárek, 1985. "Modern approach to the classification system of cyanophytes 1-Introduction". Archiv für Hydrobiologie, Supplement., 71: 291-302.

1988. "Modern approach to the classification system of cyanophytes. 3- Oscillatoriales". Archiv für Hydrobiologie, Supplement., 80: 327-472.

Arzate-Cárdenas, M.A.; R. Olvera-Ramírez, y F. Martínez-Jerónimo, 2010. "Microcystis toxigenic strains in urban lakes: a case of study in Mexico City". Ecotoxicology, 19: 1157-1165.

Ayala-Pérez, L.A.; O.A. Avilés Alatriste, y J.L. Rojas-Galavíz, 1998. "Estructura de la comunidad de peces en el sistema Candelaria-Panlau, Campeche, México". Rev. Biol. Trop., 46(3): 763-774.
Banker, R.; B. Teltsch, A. Sukenik, y S. Carmeli, 2000. "7-Epicylindrospermopsin, a toxic minor metabolite of the cyanobacterium Aphanizomenon ovalisporum from Lake Kinneret, Israel". J. Nat. Prod., 63: 387-389.

Becerra-Cabañas, L.E., 2009. “Catálogo fitoplactónico de los Lagos del Dique, Municipio de Xalapa, Veracruz, México". Tesis de licenciatura. Facultad de Biología de la Universidad Veracruzana.

Beltrán, E.C., y B.A. Neilan, 2000. “Geographical Segregation of the NeurotoxinProducing Cyanobacterium Anabaena circinalis". Appl. Environ. Microbiol., 66(10): 4468-74.

Berry, J.P.; E. Lee, K. Walto, A.E. Wilson, y F. Bernal-Brooks, 2011. "Bioaccumulation of Microcystins by fish associated with a persistent Cyanobacterial Bloom in Lago de Patzcuaro (Michoacan, Mexico)". Environ. Toxicol. Chem., 30(7): 1621-1628.

Bostock, P.D., y A.E. Holland, 2010. Census of the Queensland Flora. (Eds.). Queensland Herbarium Biodiversity and Ecosystem Sciences, Department of Environment and Resource Management. Brisbane. 324 pp.

Bowling, L.C., y P.D. Baker, 1996. "Major cyanobacterial bloom in the BarwonDarling River, Australia, in 1991, and underlying limnological conditions". Mar. Freshw. Res., 47: 643-657.

Bozada, L., y M. Páez, 1986. La fauna acuática del litoral. (Eds.). Serie medio 
ambiente en Coatzacoalcos. Volumen XIV. Centro de Ecodesarrollo, México DF. 120 pp.

Bricker, S.; C. Clement, S. Frew, M. Harmon, M. Harris, y D. Pirhalla, 1997. "National Oceanic and Atmospheric Administration (NOAA)". En NOAA's Estuarine Eutrophication Survey, Volume 4: Gulf of Mexico Region. Silver Spring, MD: Office of Ocean Resources Conservation and Assessment. 77 pp.

Campos, V.; S. Lisperguer, J. Weckesser, A. Vera, y D. Muñoz, 2005. "Cianobacterias y riesgos potenciales de toxicidad en aguas continentales de Chile". Boletín Micológico, 20: 73-81.

Carmichael, W.W., y I.R. Falconer, 1993. "Diseases related to freshwater bluegreen algal toxins, and control measures", en Falconer, IR (Eds.). Algal toxins in seafood and drinking water. Academic Press Ltd. London. 187209 pp.

Carneiro, R.L.; M.E.V. Santos, A.B.F. Pacheco, y S.M.F.O. Azevedo, 2009. "Effects of light intensity and light quality on growth and circadian rhythm of saxitoxins production in Cylindrospermopsis raciborskii (Cyanobacteria)". J. Plan. Res., 31(5): 481-488.

Carrasco, D.; E. Moreno, T. Paniagua, C. de Hoyos, L. Wormer, D. Sanchis, S. Cirés, D. Martín-del-Pozo, G.A. Codd, y A. Quesada, 2007. "Anatoxin-a occurrence and potential cyanobacterial anatoxin-a producers in Spanish reservoirs". J. Phycol., 43: 1120-1125.

Carvalho, M.; M.S.F. Gianesella, y F.M.P. Saldanha-Corrêa, 2008. "Trichodesmium erythraeum bloom on the continental shelf off Santos, Southeast Brazil’. Braz. J. Oceanog., 56(4): 10-12.

Carvajal, G., 2011. "Occurrencia de floraciones de cyanobacterias toxicas en cuerpos de agua dulce, Argentina. Estudio de campo y análisis de datos". Tesis de maestría. Cátedra de Toxicología de la Facultad de Ciencias Exactas, Laboratorio Científico de Ficología, Facultad de Ciencias Naturales y Museo de la Universidad Nacional de La Plata. 185 pp.

Chomérat, N.; R. Garnier, C. Bertrand, yA. Cazaubon, 2007. "Seasonal succession of cyanoprokaryotes in a hypertrophic oligo-mesohaline lagoon from the South of France". Est. Coast. Shelf Sci., 72: 591-602.

Chorus, I., y J. Bartram, 1999. Toxic cyanobacteria in water: A guide to their public health consequences, monitoring and management. E y FN Spon, London. 416 pp.

Cirés-Gómez, S., y A. Quesada de Corral, 2011. Catálogo de cianobacterias planctónicas potencialmente tóxicas de las aguas continentales españolas. (Eds.). Madrid: Ministerio de Medio Ambiente y Medio Rural y Marino, España. 85 pp.

Cortés-Altamirano, R., y S. Licea-Durán, 1999. "Florecimientos de microalgas 
Muciño-Márquez,R.E.etal.:Cianofitasdesistemasfluvio-lagunaresPom-AtastayPalizadadel Este,Lag.deTérminos,Camp.,Méx.

nocivas en estanques para cultivo semiintensivo de camarón en México". Rev. Latinoam. Microb., 41: 157-166.

De la Lanza-Espino, G., y G. Gómez Rodríguez, 2005. "Analysis of the short term geo-environmental evolution of an endorrey basin using satellite images: the case of Tecocomulco Lagoon, Mexico". Invest. Geogr. Bol. Inst. Geogr., 58: 66-79.

Dortch, Q., 2002. "Faculty Focus". Lumcon News, 2(2): 4-5.

Echenique, R.O., y A. Aguilera, 2009. “Capítulo 3: Floraciones de Cyanobacteria toxígenas en la República Argentina: antecedentes", en Giannuzzi, L., A. Colombi, T. Pruyas, A. Aun, M. Rujana, M. Falcione y J. Zubieta. Cianobacterias y cianotoxinas: identificación, toxicología, monitoreo y evaluacion de riesgo. Corrientes, Moglia Impresiones. 238 pp.

Ersanli, E., y A. Gönülol, 2006. "A study on the phytoplankton of Lake Simenit, Turkey". Cryptogamie Algologie, 27: 289-305.

Forti, A., 1911. "Diagnoses myxophycearum novarum". Atti Mem. Accad. Agric. Sci. Lettere, Arti. Commer. Ver., 12: 3-5.

Gárate-Lizárraga, I., y R.E. Muciño-Márquez, 2012. "Blooms of Trichodesmium erythraeum and $T$. thiebautii (Cyanobacteria, oscillatoriales) in the Bahía de La Paz, Gulf of California". CICIMAR Oceánides, 27(1): 61-64.
Gómez-Aguirre, S., 1974. "Reconocimientos estacionales de hidrología y plancton en la laguna de Términos, Campeche, México (1964-1965)". An. Centro. Cienc. del Mar y Limnol. Univ. Nal. Autón. México, 1: 61-82.

Gomont, M., 1892. "Monographie des Oscillariées (Nostocacées homocystées)". Ann. Sci. Nat. Bot., 16: 91-264.

Guiry, M.D., y G.M. Guiry, 2012. “AlgaeBase. World-wide electronic publication". National University of Ireland, Galway. http://www.algaebase.org/.

Gutiérrez-Estrada, M.; V.M. Malpica-Cruz, y J. Martínez-Reyes, 1982. "Geomorfología y sedimentos recientes del sistema lagunar Atasta-Pom, Campeche, México". An. Inst. Cienc. del Mar y Limnol. Univ. Nal. Autón. México., 9: 89-100.

Hasle, G.R., 1978. "Using the inverted microscope", en: Sournia, A. (Eds.) Phytoplankton Manual. UNESCO, París.

Hašler, P., y A. Poulíčková, 2010. “Diversity, taxonomy and autecology of autochtonous epipelic cyanobacteria of the genera Komvophoron and Isocystis (Borziaceae, Oscillatoriales)". Biologia, 65(1): 7-16.

Hawkins, P.R.; M.T.C. Runnegar, A.R.B. Jackson, y I.R. Falconer, 1985. "Severe hepatotoxicity caused by the tropical cyanobacterium (blue-green alga) Cylindrospermopsis raciborskii (Woloszynska) Seenaya and Subba Raju 
isolated from a domestic water supply reservoir". Appl. Environ. Microbiol., 1292-1295.

Herrera-Silveira, J.A., y S.A. Morales-Ojeda, 2009. "Evaluation of the health status of a coastal ecosystem in southeast Mexico: Assessment of water quality, phytoplankton and submerged aquatic vegetation". Mar. Pollut. Bull., 59: 72-86.

John, D.M.; B.A. Whitton, y A.J. Brook, 2002. The Freshwater Algal Flora of the British Isles. Cambridge University Press, UK.

Jones, W.W, y S. Sauter, 2005. Distribution and Abundance of Cylindrospermopsis raciborskii in Indiana Lakes and Reservoirs. Schoool of Public and Environmental Affairs, Indiana University. $54 \mathrm{pp}$.

Jozwiak, T., H. Mazur-Marzec, y M. Plinski, 2008. "Cyanobacterial blooms in the Gulf of Gdan'sk (southern Baltic): The main effect of eutrophication". Oceanol. Hydrobiol.Stud., 37: 115121.

Kling, H.J.; L.D. Findlay, y J. Komárek, 1994. "Aphanizomenon schindleri sp.nov.: a new nostocacean cyanoprokaryote from the Experimental Lakes Area, northwestern Ontario". Can. J. Fish. Aquat. Sci., 51(10): 2267-2273.

Kokocinski, M., y J. Soininen, 2012. "Environmental factors related to the occurrence of Cylindrospermopsis raciborskii (Nostocales, Cyanophyta) at the north-eastern limit of its geographical range". Eur. J. Phycol., 47(1): 12-21.

Komárek, J., 1974. “The morphology and taxonomy of crucigenoid algae (Scenedesmaceae, Chlorococcales)". Archiv. Für. Protistenkunde., 116: 1-74.

2005. "Phenotype diversity of the heterocytous cyanoprokaryotic genus Anabaenopsis". Czech. Phycology. Olomouc, 5: 1-35.

Komárek, J., y H. Kling, 1991. "Variation in six planktic cyanophyte genera in Lake Victoria (East Africa)". Arch. Hydrobiol. Algolog. Stud., 61: 24-45.

Komárek, J., y K Anagnostidis, 1999. “Cyanoprokaryota 1. Teil: Chroococcales”, en Ettl, H., G. Gärtner, H. Heynig, y D. Mollenhauer. (Eds). Süsswasserflora von Mitteleuropa 19/1, Gustav Fischer, Jena-Stuttgart-Lübeck-Ulm. 548 pp.

2005. “Cyanoprokaryota 2". Teil/ 2nd Part: Oscillatoriales, en Büdel, B., L. Krienitz, G. Gärtner, y M. Schagerl. (Eds). Süsswasserflora von Mitteleuropa 19/2, Elsevier/Spektrum, Heidelberg. 759 pp.

Komárek, J., y J. Komárková-Legnerová, 2002. "Preparation of the World Cyanobacterial Database", en Shimura, J., K.L. Wilson, y D. Gordon. (Eds). Proc. 2nd Internat. Workshop "Species 2000”, Res. Rep. NIES, Japan, 171: 64-69.

Komárek, J., y J. Komárková, 2003. “Phenotype diversity of the cyanoprokaryotic 
Muciño-Márquez,R.E.etal.:Cianofitasdesistemasfluvio-lagunaresPom-AtastayPalizadadel Este,Lag.deTérminos,Camp.,Méx.

genus Cylindrospermopsis (Nostocales); review 2002". Czech. Phycology. Olomouc, 3: 1-30.

Komárek, J., y E Zapomělová, 2007. "Planktic morphospecies of the cyanobacterial genus Anabaena=subg. Dolichospermum-1. part: coiled types". Fottea. Czech. Phycology. Olomouc, 7(1): 1-31.

Komárková-Legnerová, J., y R. Tavera, 1996. "Cyanoprokaryota (Cyanobacteria) in the phytoplankton of Lake Catemaco (Veracruz, Mexico)". Arch. Hydrobiol. Algolog. Stud., 83: 403-422.

2003. "Steady state of phytoplankton assemblage in the tropical Lake Catemaco (Mexico)". Hydrobiologia, 502: 187-196.

Komárková, J., J. Jezberová, O. Komárek, y E. Zapomêlová, 2010. "Variability of Chroococcus (Cyanobacteria) morphospecies with regard to phylogenetic relationships". Hydrobiologia, 639: 69-83.

Kützing, F.T., 1845. Phycologia germanica, d.i. Deutschlands Algen in bündigen Beschreibungen. Nebst einer Anleitung zum Untersuchen und Bestimmen dieser Gewächse für Anfänger. Nordhausen: W. Köhne. 1-340. pp.

Kützing, F.T., 1849. "Species algarum”, en Guiry, M.D. y G.M. Guiry, 2013. AlgaeBase. World-wide electronic publication, National University of Ireland, Galway. http://www.algaebase.org.
Lanzarot-Freudenthal, Ma.P., 2007. Cianobacterias tóxicas y mortandades en masa de fauna salvaje en las Marismas de Doñana. (Eds.). Departamento de Producción Animal, Genética, Facultad de Veterinaria, Universidad Complutense de Madrid, España.

Leão, P.N.; M.T.S.D. Vasconcelos, y V.M. Vasconcelos, 2009. "Allelopathy in freshwater cyanobacteria". Crit. Rev. Microbiol., 35(4): 271-282.

Legaria, M.L., 2003. "Dinámica del fitoplancton y su relación con variables físico-químicas en la laguna costera La Mancha, Ver., Méx". Tesis de licenciatura. Facultad de Biología, Universidad Veracruzana, Xalapa, Veracruz.

Lemmermann, E., 1904. "Das Plankton schwedischer Gewässer". Arkiv. Bot., 2(2): 1-209.

Licona, S.M, 2003. "Diversidad fitoplanctónica del río Sedeño, tramo Plan Rincón del Sedeño-Col. 6 de enero". Tesis de licenciatura. Facultad de Biología, Campus Xalapa, Universidad Veracruzana, Xalapa, Veracruz.

López-Adrián, S.J., y R.C. Barrientos-Medina, 2005. "La colección de microalgas dulceacuícolas de la península de Yucatán”. Universidad Autónoma de Yucatán, Mérida.

López-Adrián, S.J., y L.A. Catzim-Cruz. 2010. Parte II. "Biodiversidad". Capítulo 4. Especies. Microalgas dulceacuícolas, en Durán R. y M.E. Méndez. 
Biodiversidad y desarrollo humano en Yucatán. Anexo II. (Eds.). Centro de Investigación Científica de Yucatán/ Programa de las Naciones Unidas para el Desarrollo/Comisión Nacional para el Conocimiento y Uso de la Biodiversidad, México, DF.

Madigan, M.T.; J.M. Martinko, y J. Parker, 2004. Brock: Biología de los Microorganismos, $8^{\mathrm{a}}$ ed., Prentice Hall Madrid.

Meichtry-Zaburlín, N.; S. Irmgard Martens, y V. Llano, 2009. "Cianobacteria planctónica: su impacto en ambientes acuáticos continentales. Descripción de los géneros más frecuentes", pp.1723, en Cianobacterias y Cianotoxinas: identificación, toxicología, monitoreo y evaluación de riesgo. Buenos Aires, Argentina.

Meyen, 1839. Meyen, F.J.F. (1839). Neues system der pflanzen-physiologie. Dritter band. pp. i-x, 1-627, Plates $\mathrm{X}-\mathrm{XV}$. Berlin: Haude und Spenersche Buchandlung (S.J. Joseephy).

Mihali, T.; R. Kellmann, y B. Neilan, 2009. "Characterisation of the paralytic shellfish toxin biosynthesis gene clusters in Anabaena circinalis AWQC131C and Aphanizomenon sp. NH-5". BMC. Biochem., 10: 8.

Moreno-Ruiz, J.L., 2000. "Fitoplancton", en De la Lanza, G., S. Hernández y J.L. Carbajal. (comps.), Organismos indicadores de la calidad del agua y de la contaminación (bioindicadores). Semarnap/Plaza y Valdés, México. 43-108 pp.
Moreno-Ruiz, J.L., 2005. "Fitoplancton", en Bueno-Soria J.S. y F. Álvarez-Noguera. (Eds.). Biodiversidad de Tabasco. Instituto de Biología, UNAM/CONABIO, México DF, México.

Muciño-Márquez, R.E.; Ma.G. FigueroaTorres, y A. Esquivel-Herrera, 2011. "Variación nictemeral de la comunidad fitoplanctónica, en la boca de la laguna costera de Sontecomapan, Veracruz". CICIMAR Oceánides, 26(1): 19-31.

Nägeli, C., 1849. "Gattungen einzelliger Algen, physiologisch und systematisch bearbeitet". Denkschr. d. Schweiz. Naturf. Gsll., 10(7): 1-139.

Namikoshi, M.; T. Murakami, M.F. Watanabe, T. Oda, J. Yamada, H. Tsu-Nagai, y S. Oishi, 2003. "Simultaneous production of homoanatoxin-a, anatoxin-a, an non-toxic 4-hydroxyhomoanatoxin-a by the cyanobacterium Raphidiopsis mediterranea Skuja". Toxicon, 42(5): 533-538.

Novelo, E., 2011. "Cyanoprokaryota". Fascículo 90 Serie: Flora del Valle de Tehuacán-Cuicatlán. (Eds.) Medina, R. Instituto de Biología, Universidad Nacional Autónoma de México, 96 pp.

Okolodkov, Y.B., y R. Blanco Pérez, 2011. "Diversidad de microalgas marinas y de aguas salobres", en La Biodiversidad en Veracruz: Estudio de Estado, vol. 2, Publisher: CONABIO, Gobierno del Estado de Veracruz, Universidad Veracruzana, Instituto de Ecología.

Oliva-Martínez, M.G.; A. Rodríguez-Rocha, A. Lugo-Vázquez, y M.R. Sánchez- 
Muciño-Márquez,R.E.etal.:Cianofitasdesistemasfluvio-lagunaresPom-AtastayPalizadadel Este,Lag.deTérminos,Camp.,Méx.

Rodríguez, 2008. “Composición y dinámica del fitoplancton en un lago urbano hipertrófico". Hidrobiológica, 18(1): 752-761.

Osorio-Sánchez, J.J., y R. López-Pérez, 2005. "Diversidad y distribución del fitoplancton de la laguna El Balsón, Tabasco, México". Tesis de licenciatura. Ciencias Biológicas. Universidad Juárez Autónoma de Tabasco.

Otaño, S.H., 2009. "First report of the cyanobacterium Aphanizomenon schindleri (Nostocales, Cyanophyceae) in River Uruguay, Argentina”. Algological Studies, 131: 35-42.

Pérez, D.S.; A.L. Soraci, y M.O. Tapia, 2008. "Cianobacterias y cianotoxinas: rol de las microcistinas en la salud humana y animal y su detección en muestras de agua". Analecta Vet., 28(1): 48-56.

Pérez-Barrón, M.I., 1991. “Composición fitoplanctónica en el bajo del río Coatzacoalcos en un ciclo anual (19871988)". Tesis de licenciatura. Facultad de Biología, Campus Xalapa, Universidad Veracruzana, Xalapa. Ver.

Pollingher, U.; O. Hadas, Y.Z. Yacobi, T. Zohary, y T. Berman, 1998. "Aphanizomenon ovalisporum (Forti) in Lake Kinneret, Israel”. J. Plankton Res., 20: 1321-1339.

Prescott, GW., 1962. Algae of the Western Great Lakes Area. (Eds.). Brown Company Publishers. USA.

Quesada, A.; E. Moreno, D. Carrasco, T. Paniagua, L. Wormer, C. de Hoyos, y A.
Sukenik, 2006. "Toxicity of Alphanizomenon ovalisporum (Cyanobacteria) in a Spanish water reservoir". Eur. J. Phycol., 41: 39-45.

Quesada-Corral, A.; D. Carrasco, y S. Cirés, 2006. "Cianobacterias en aguas de consumo humano y de recreo. Un problema de todos". Ponencia en Centro de Estudios y Experimentación de Obras Públicas (CEDEX).

Ramírez-García, P.; E. Martínez-Romero, M.D. Martínez-Salgado, y C.A. Eslava-Campos, 2004. Cianobacterias, microorganismos del fitoplancton y su relación con la salud humana. Instituto Nacional de Ecología, México.

Rodríguez-Palacio, M.C.; S. Álvarez-Hernández C. Lozano-Ramírez, G. De Lara-Isassi, y G. Vázquez Hurtado, 2008. "Cepas de microalgas dulceacuícolas y cianobacterias cultivadas, del lago de origen volcánico Chalchoapan, Veracruz". I Congreso latinoamericano de biotecnología ambiental, II Congreso latinoamericano de biotecnología algal, V Symposium internacional sobre bioprocesos más limpios y desarrollo sustentable, Xalapa, Veracruz.

Rojas-Galaviz, J.L.; F. Vera-Herrera, A. Yáñez-Arancibia, y Jr.J.W. Day, 1990. "Ecosystem dynamics of the Palizada River Delta, Mexico: The role of the dual riverine/tidal subsidy in a tropical tidal freshwater environment". Estuaries, 42: 663-672.

Sánchez-Chávez, J.J.; L.A. Bravo-Inclán, A.C. Tomasini-Ortiz, R. González- 
Villela, M.A. Córdova-Rodríguez, y R.S. Villalobos-Hernández, 2011. "Monitoreo de la calidad del agua del lago y de las descargas. Subcoordinación Hidrobiología y Evaluación Ambiental". Coordinación Tratamiento y Calidad del Agua (Proyecto final núm. TC-0847). Recuperación Ambiental de la Cuenca del Lago de Pátzcuaro. Fundación Gonzalo Río Arronte, I.A.P. Instituto Mexicano de Tecnología del Agua.

Skulberg, O.M.; W.W. Carmichael, G.A. Codd, y R. Skulberg, 1993. "Taxonomy of toxic Cyanophyceae (Cyanobacteria)", en: Algal toxins in seafood and drinking water. Academic Press.

Suárez-Caabro, J.A., y S. Gómez-Aguirre, 1965. "Observaciones sobre el plancton de la Laguna de Términos, Campeche, México". Bull. Mar. Sci., 15: 1073-1119.

Tomasini-Ortiz, A.C.; G. Moeller-Chávez, J.J. Sánchez-Chávez, y L.A. Bravo-Inclán, 2012. "Cianobacterias y cianotoxinas en el lago de Pátzcuaro, Michoacán, México". Revista AIDIS, 5(2): 93-101.

Tomitani, A.; A.H. Knoll, C.M. Cavanaugh, y T. Ohno, 2006. "The evolutionary diversification of cyanobacteria: Molecular-phylogenetic and paleontological perspectives". Proc. Natl. Acad. Sci. USA, 103: 5442-5447.

Vasconcelos, V.; A. Martins, M. Vale, A. Antunes, J. Azevedo, M. Welker, O. López, y G. Montejano, 2010. "First report on the occurrence of micro- cystins in planktonic cyanobacteria from Central Mexico". Toxicon, 56(3): 425-431.

Vázquez, G., y L. Legaria, 2006. "Las algas". En: Moreno-Casasola, P. Entornos veracruzanos: La costa de la Mancha. (Eds.). Instituto de Ecología, A.C., Xalapa, Veracruz, México.

Vázquez, G., y R. Blanco-Pérez, 2011. "Microalgas de agua dulce de Veracruz", en: La biodiversidad en Veracruz: estudio de estado". vol. II. 77-88 pp. Comisión Nacional para el Conocimiento y Uso de la Biodiversidad, Gobierno del Estado de Veracruz, Universidad Veracruzana, Instituto de Ecología, A.C.

Vázquez, G.; S. Jiménez, M.E. Favila, y A. Martínez, 2005. "The relationship between seasonal dynamics of phytoplankton and the cyanobacterial dominance in a eutrophic crater lake (Los Tuxtlas, México)". Ecoscience, 12(4): 485-493.

Vázquez, G.; E. Díaz Pardo, I. Doadrio, A. De Sostoa, R. Madrigal, L. Legaria, R. Landgrave, G. Rangel, y A. Gutiérrez, 2002. Diversidad y distribución de las comunidades de fitoplancton y peces de ríos y lagunas del volcán San Martín de la reserva de la biósfera Los Tuxtlas. Instituto de Ecología A.C. Informe final SNIB-CONABIO. Proyecto No. S022. México, DF.

Vázquez, G.; E. Díaz-Pardo, A. GutiérrezHernández, I. Doadrio, y A. de Sostoa. 2004. "Los ríos y los lagos", en: Los Tuxtlas, Guevara, S., J. Laborde y G. 
Muciño-Márquez,R.E.etal.: Cianofitasdesistemasfluvio-lagunaresPom-AtastayPalizadadel Este,Lag.deTérminos, Camp., Méx.

Sánchez-Ríos. (Eds.). Unión EuropeaInecol.

Villarauz, K., 2006. “Dinámica espacial y temporal del epiliton en ríos de la cuenca alta del río La Antigua, Ver.”. Tesis de licenciatura. Benemérita Universidad Autónoma de Puebla, Puebla.

Yáñez-Arancibia, A.; F. Amezcua-Linares, y J.W. Day Jr., 1980. "Fish community structure and function in Terminos Lagoon, a tropical estuary in the southern Gulf of Mexico", en: Kennedy, V.S. (Eds.). Estuarine perspectives. Academic, Nueva York.

Wacklin, P.; L. Hoffmann, y J. Komárek, 2009. "Nomenclatural validation of the genetically revised cyanobacterial genus Dolichospermum (Ralfs ex Bornet et Flahault) comb. Nova". Fottea, 9(1): 59-64.
Walker, D., 2004. "Water Quality Monitoring Report”. Issue 1. Environmental Research Lab, University of Arizona.

West, W., y G.S. West, 1894. "New British freshwater algae". J.R. Microsc. Soc., $1-17$.

Whitton, B., y M. Potts, 2000. "Introduction to cyanobacteria". (Eds.). The ecology of cyanobacteria. Kluwer Academic, Dordrecht, Países Bajos.

Wu, Z.; J. Shi, S. Lin, y R. Li, 2010. "Unraveling molecular diversity and phylogeny of Aphanizomenon (Nostocales, Cyanobacteria) strains isolated from China". J. Phycol., 46(5): 1048-1058.

Recibido: 4 junio 2013. Aceptado: 11 junio 2014. 

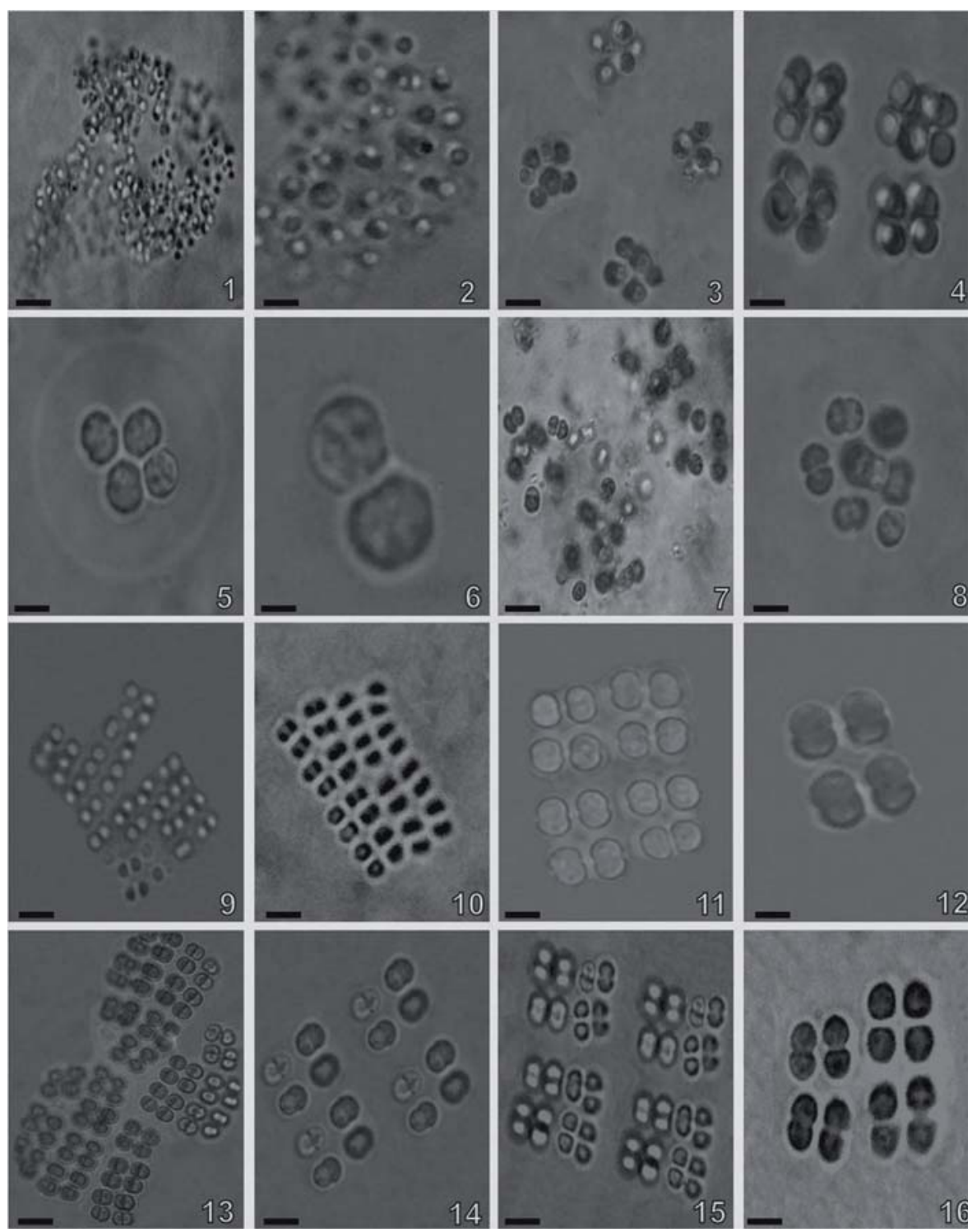

Lámina 1: 1-2) Aphanocapsa elachista, 3-4) Chroococcus dispersus, 5-6) C. minutus, 7-8) Limnococcus limneticus, 9-10) Merismopedia convoluta, 11-12) M. elegans, 13-14) M. glauca, 15-16) M. punctata. Escala10 $\mu \mathrm{m}$. 
Muciño-Márquez,R.E.etal.:Cianofitasdesistemasfluvio-lagunaresPom-AtastayPalizadadel Este,Lag.deTérminos,Camp.,Méx.
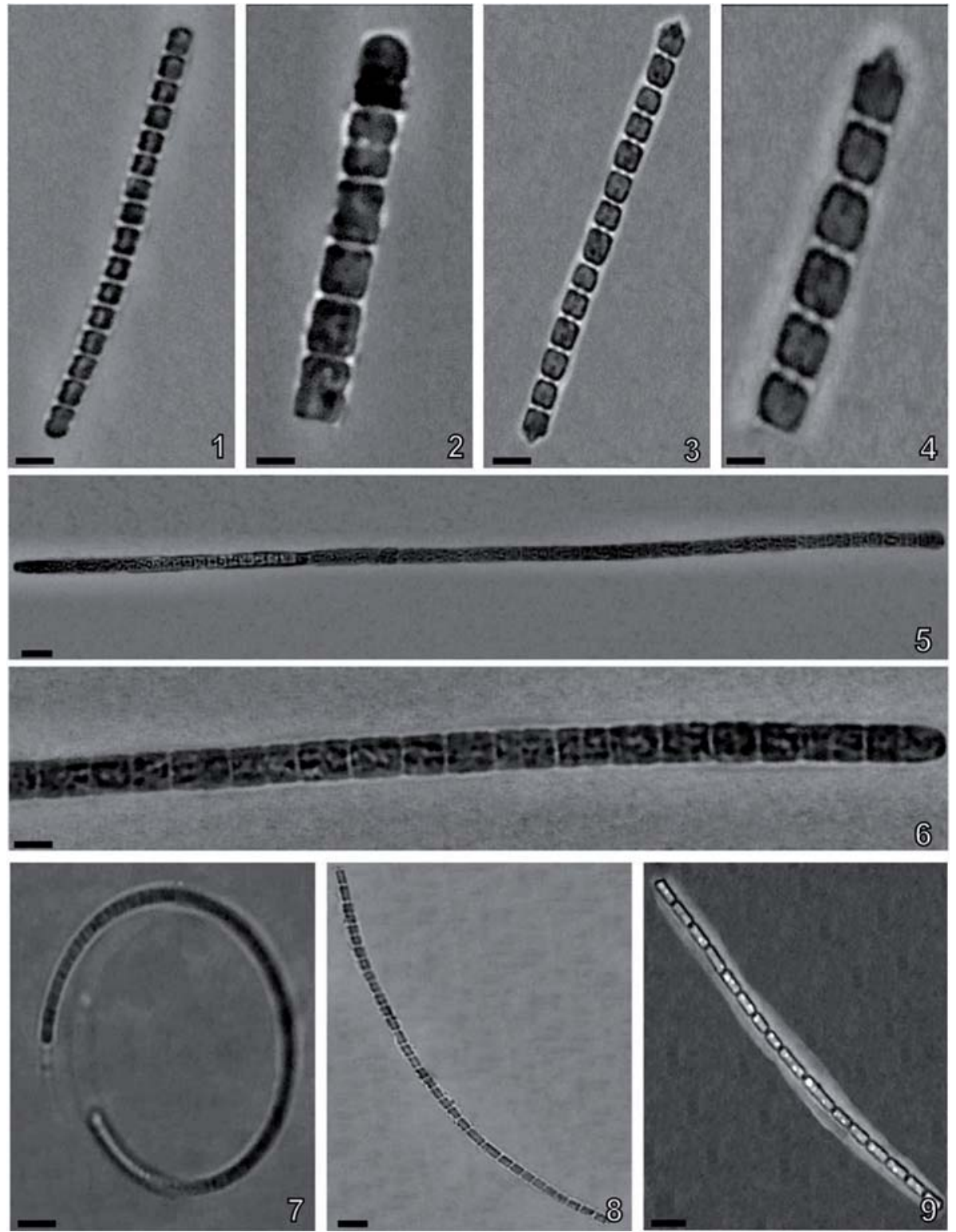

Lámina 2: 1-2) Komvophoron minutum, 3-4) Komvophoron sp, 5-6) Phormidium retzii, 7) Planktolyngbya circumcreta, 8) Pseudanabaena catenata, 9) Pseudanabaena limnetica. Escala $10 \mu \mathrm{m}$. 

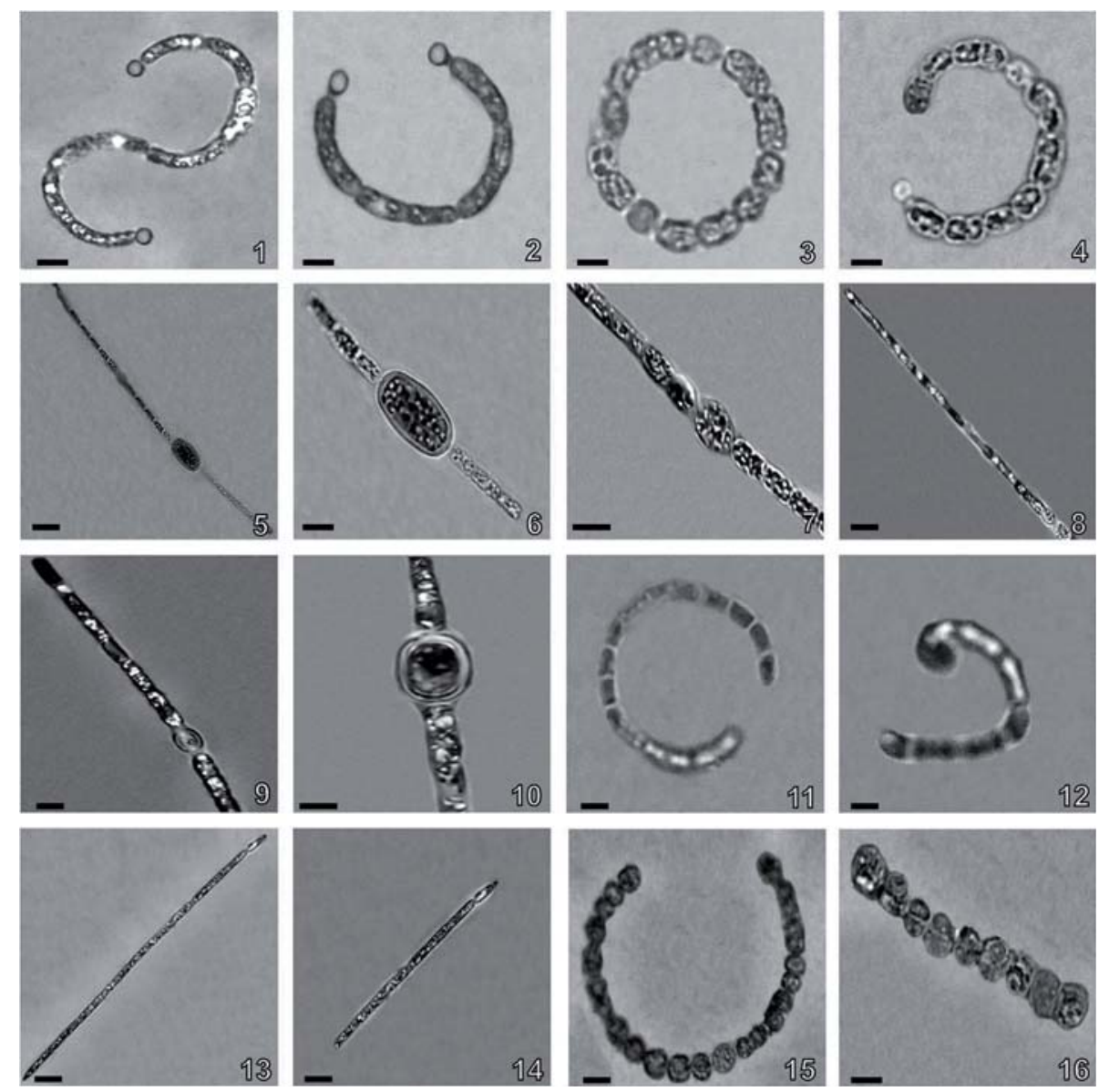

Lámina 3: 1-2) Anabaenopsis circularis, 3-4) A. elenkinii, 5-6) Anabaena sp, 7-8) Aphanizomenon cf. ovalisporum, 9-10) A. schindleri, 11-12) Cylindrospermopsis taverae, 13-14) C. cuspis, 15-16) Dolichospermum circinalis. Escala $10 \mu \mathrm{m}$. 